\title{
Influence des acides gras polyinsaturés n-3 et des antioxydants alimentaires sur les acides gras de la viande et la lipoperoxydation chez le bovin en finition
}

\author{
Dominique BAUCHART ${ }^{1}$ \\ Mylène GOBERT ${ }^{1}$ \\ Mihaela $\mathrm{HABEANU^{1,2 }}$ \\ Émilie PARAFITA ${ }^{3}$ \\ Dominique GRUFFAT ${ }^{1}$ \\ Denys DURAND ${ }^{1}$ \\ 1 Inra,
Unité de recherche sur les herbivores,
Centre de recherche de Clermont-Ferrand
Theix,
63122 Saint-Cenès-Champanelle,
France
$<$ dominique.bauchart@clermont.inra.fr'
2 IBNA,
Balotesti,
Roumanie
${ }^{3}$ ADIV,
63000 Clermont-Ferrand,
France
}

Le consommateur est de plus en plus attentif et averti du lien putatif entre les acides gras (AG) consommés apportés par la viande et la santé de l'Homme. Un apport accru d'acides gras polyinsaturés de la famille $n-3$ (AGPI $n-3$ ) par les produits animaux, associé à un rapport AGPI n-6/AGPI n-3 égal ou inférieur à 5 , serait bénéfique à la santé humaine (Afssa, 2001). En effet, de nombreux travaux menés sur l'Homme et différents modèles animaux pour l'Homme montrent que les AGPI n-3 seraient essentiels pour la structure des membranes et les fonctions associées (notamment au niveau du cerveau et de la rétine) et exerceraient des effets protecteurs vis-à-vis de l'hypertension et des maladies cardiovasculaires, de certains cancers (du sein notamment), de diabètes, de désordres neuropsychiatriques et de maladies autoimmunes (Narayan et al., 2006). Puisque la supplémentation des rations par de la graine de lin extrudée chez le bovin en finition favorise

\begin{abstract}
The aim of this study was to analyze, on beef lipids and FA and on beef colour, the impact of extruded linseed (rich in n-3 PUFA) given alone or with rapeseed (rich in n-3 and n-6 PUFA and in 18:1 $n-9)$ in association or not with antioxidants given to Normand (fat breed) cull cows during the finishing period. Dietary linseeds significantly increased proportions of 18:3n-3 (+56 and $+36 \%$ respectively), total trans 18:1 (+66 and $+105 \%)$; and of 9cis, 11 trans 18:2 (CLA) (+50 and $+41 \%)$ in LT muscle of which 18:3n-3 and CLA are known to be beneficial for the human health. Addition of antioxidants (vitamin E plus plant extracts rich in polyphenols, PERP) reinforced the stimulating effect of lipid supplements on proportions of the three considered FA (18:3 n-3, total trans 18:1, 9cis, 11trans 18:2) in total lipids of both muscles. Dietary n-3 PUFA reduced the resistance capacity of plasma against lipoperoxidation $(-11 \%)$ favouring peroxidized products formations such as conjugated dienes $(\times 1.75)$ and malonedialdehyde $(M D A, \times 2)$. Intensity of beef lipoperoxidation (estimated by MDA level) tended to be higher in meats packaged under modified atmosphere rich in oxygen (70\%) in the linseed group $(2.96 \mu \mathrm{g} / \mathrm{g}$ of tissue) compared to that of the control group $(2.19 \mu \mathrm{g} / \mathrm{g}$ of tissue, $\mathrm{P}=0.1)$. Dietary vitamin E provided with PERP effectively protected beef against lipoperoxidation, even in beef packagings with $\mathrm{O}_{2}$. Beef under air packaging from cows given the linseed supplement had a higher redness value than that from control cows. In the case of animals given lipid supplemented diets and submitted to an emotional stress, beef packaged under air or modified atmosphere rich in $\mathrm{O}_{2}$ had a lower redness than that of the same animals given lipids associated to antioxidant supplements. We concluded that the dietary strategy combining addition of n-3 PUFA and of the antioxidant mixture in diets would avoid major risks of lipoperoxidation and alteration of colour in beef products whatever: 1) the conditions of meat ageing and packaging (especially those with contact with oxygen); 2) the degree of emotional stress of animals just prior slaughter.
\end{abstract}

Key words: beef, $n-3$ fatty acids, lipoperoxidation, antioxidants, finishing cows

le dépôt des AGPI n-3 dans la viande crue comme cuite (Bauchart et al., 2005 ; Normand et al., 2005), celle-ci peut être une source supplémentaire d'AGPI n-3 en nutrition humaine.

Nous présentons ici plusieurs résultats majeurs du Programme Lipivimus de l'Agence nationale de la recherche (2007-2009) concernant les effets des AGPI n-3 et d'antioxydants (vitamine $E$ [vit E], extraits de plantes riches en polyphénols [EVRP]) alimentaires sur les lipides circulants et tissulaires chez le bovin en finition. Les effets de ces composés ajoutés à la ration de vaches de réforme normandes en finition portent sur :

- les modifications des caractéristiques de distribution et la teneur des AG du muscle Longissimus thoracis (LT) ;

- la sensibilité relative des lipides à la lipoperoxydation dans le plasma et les muscles à l'abattage, et dans les viandes après application de divers modes de maturation et de conditionnement.

Ce programme de recherche a pour objectifs majeurs de préciser l'impact de facteurs liés à l'animal (âge, sexe, génotype) et à son alimentation (composition de la ration de base, différentes sources de suppléments lipidiques et en antioxydants), et leurs interactions expliquant la variabilité des caractéristiques lipidiques (teneur en lipides, composition en AG) des muscles squelettiques en relation avec les caractéristiques de qualité des viandes.

Les effets des suppléments lipidiques et des suppléments en antioxydants sont comparés en vue de déterminer des conditions d'alimentation performantes et durables pour une production de viandes de meilleures qualités nutritionnelles et sensorielles. Ces recherches visent, de plus, à rechercher le meilleur équilibre entre un dépôt accru d'AGPI n-3 dans les viandes et l'absence d'effets délétères sur la santé de l'ani- 
mal et la qualité des lipides de la viande. En effet, un effet néfaste de la lipoperoxydation peut être favorisé non seulement par la haute sensibilité des AGPI n-3 aux attaques d'espèces réactives de l'oxygène $\left(\mathrm{O}_{2}\right)$ au cours d'un stress oxydant, mais aussi par un déséquilibre du statut de l'animal en antioxydants alimentaires ou endogènes. De plus, l'intensité de lipoperoxydation dans la viande peut être potentiellement stimulée par un stress émotionnel et/ou physique des animaux, juste avant l'abattage, ou par certains traitements technologiques appliqués aux viandes tels que des conditionnements en présence $d^{\prime} \mathrm{O}_{2}$.

\section{Effets des AGPI n-3 et des antioxydants alimentaires sur les AG de la viande bovine}

Les lipides influencent fortement les valeurs nutritionnelles-santé et les qualités sensorielles des viandes, particulièrement celles des ruminants (Geay et al., 2001). Les facteurs liés au type d'animal et à son alimentation peuvent moduler, particulièrement au cours de la période de finition, les caractéristiques des lipides de la viande telles que :

- leur concentration en relation avec le développement du tissu adipeux intramusculaire ; - la distribution des classes lipidiques quantitativement majeures (triglycérides [TG], et à un degré moindre, phospholipides [PL]) et mineures (cholestérol sous forme libre et estérifiée, diglycérides et AG libres);

- leur composition en AG à chaîne longue.

Ainsi, I'impact des sources alimentaires riches en AGPI n-3 (herbe fraîche, graines oléagineuses, huiles de poisson) en période de finition a été largement décrit chez le jeune bovin mâle (< deux ans) dont les muscles sont relativement pauvres en dépôts gras (Geay et al., 2001 ; Scollan et al., 2005). En revanche, l'impact de ces traitements sur les AG de la viande est peu documenté chez des bovins âgés et gras, tels que cela se pratique en France avec des vaches de réforme laitières ou allaitantes soumises à un traitement alimentaire dit « de finition » riche en énergie apportée par une ration à base d'aliment concentré.

Le but de cette étude a été de comparer les effets, sur les caractéristiques des lipides (teneur tissulaire, distribution en classes de lipides) et sur la composition de leurs AG, de l'apport de graines de lin (riches en 18:3 n-3) seul à celui d'un mélange de graines de lin et de colza (riches en AGPI n-3 et n-6 et en 18:1 n-9) en association ou non avec des antioxydants alimentaires chez des vaches de réforme en finition.

\section{Aspects expérimentaux}

\section{Animaux, aliments et prélèvements}

L'expérience a été conduite sur 72 vaches de réforme normandes (race à tendance grasse) en finition pendant 100 jours (neuf traitements alimentaires, huit animaux par lot) pour analyser les qualités des viandes et les effets des interactions possibles entre les suppléments alimentaires lipidiques et en antioxydants, en situation de stress émotionnel ou non des animaux.

Les animaux ont reçu des régimes composés d'une même ration de base constituée d'aliment concentré (70 \%) et de paille (30\%), distribués seuls (régime témoin T) ou avec un supplément lipidique (40 g de lipides/kg d'aliment $\mathrm{sec}$ ) à base de graines de lin extrudées (régime L) ou du mélange de graines de colza (deux tiers) et de lin (un tiers) extrudées (régime $\mathrm{CL}$ ) apporté seul ou avec des antioxydants de type vit $E$ seule $(155 \mathrm{IU} / \mathrm{kg}$ ) [régimes LE et CLE] ou associée à un mélange $\mathrm{d}^{\prime} \operatorname{EVRP}(7 \mathrm{~g} / \mathrm{kg})$ à base de romarin, de raisin, d'agrumes et d'œillet d'Inde (régimes LEP et CLEP) (origine : Société phytosynthèse, Riom, France).

Certains animaux ont été soumis à des conditions de stress émotionnel juste avant leur abattage, consécutif à un transport seul de $15 \mathrm{~min}$ en bétaillère, suivi d'une course forcée de 30 min dans un dédale de couloirs disposés en chicane et terminé d'un transport seul de 15 min en bétaillère jusqu'à I'abattoir (traitements LS et LEPS).

Les régimes sont calculés pour être isoénergétiques et isoazotés permettant une vitesse de croissance de $1150 \mathrm{~g} / \mathrm{jour}$. Les animaux ont été abattus à poids vif et note d'état corporel $(3,0-3,5)$ similaires à l'abattoir du centre Inra de Theix. Les paramètres zootechniques (efficacité alimentaire, gain de poids vif, couleur et degré de dépôts lipidiques des carcasses, pH, température et composition des carcasses) ont été déterminés sur tous les animaux de l'expérience. Des échantillons représentatifs des muscles LT et Semitendinosus (ST) (120-150 g) ont été prélevés un jour post-mortem, puis découpés en petits cubes et congelés dans $\mathrm{N}_{2}$ liquide. Ils sont finalement réduits en poudre fine et homogène dans $\mathrm{N}_{2}$ liquide par un broyeur à lame rotative à haute vitesse, puis stockés à $-80^{\circ} \mathrm{C}$ jusqu'aux analyses des lipides et de leurs $A G$.

\section{Méthodes d'analyse des lipides et des AG de la viande}

Les lipides totaux des muscles LT et ST sont extraits à partir de $6 \mathrm{~g}$ de poudre de viande par le mélange chloroforme/méthanol 2/1 (V/ V) selon la méthode de Folch et al. en 1957, puis la concentration des lipides est déterminée par gravimétrie. Les différentes classes lipidiques sont séparées par HPLC (Kontron, Suisse) sur colonne de silice $5 \mu \mathrm{m}$ (150 mm de long; d.i. : 4,6 mm), avec un gradient ternaire de solvants, puis quantifiées par détection de diffusion de lumière (DDL, Sedere, France) selon la procédure décrite par Reynolds et Anderson (1998).

Les AG à chaîne longue sont extraits et transméthylés à partir des lipides totaux par le mélange BF3-méthanol $14 \%$. La composition centésimale détaillée des AG est déterminée par CPG et détection en ionisation de flamme (Perichrom, France) sur colonne capillaire de verre de type polaire haute résolution (100 m de long, d.i. : 0,25 mm), de type CP Sil 88 (Varian, France) avec I' $\mathrm{H}_{2}$ comme gaz vecteur. Le coefficient de réponse à la flamme de chaque AG est calculé à l'aide du mélange standard d'esters méthyliques C4-C24 (Supelco, États-Unis). Les valeurs sont exprimées en moyenne, les effets des traitements alimentaires étant analysés statistiquement par le test $t$ de Student.

\section{Résultats}

\section{Lipides et $A G$ de la viande}

\section{Distribution des classes de lipides}

Les teneurs en matière sèche et en lipides totaux sont 6 et $31 \%$ plus élevées dans le muscle LT que dans le muscle ST respectivement (données non fournies). Pour ces deux muscles, la teneur en lipides totaux varie avec celle des TG, alors que celle des PL est stable (tableau 1). Les lipides quantitativement mineurs (cholestérol libre, esters de cholestérol, diglycérides et AG libres) représentent 9,8 et $8,9 \%$ des lipides totaux des muscles LT et ST, respectivement (tableau 1). L'addition de lipides insaturés (lin, colza) dans la ration de base ne modifie pas significativement le contenu en lipides totaux et de leurs classes majeures dans les muscles. En revanche, les teneurs en AG libres et en diglycérides tendent à être plus élevées avec les régimes enrichis en lipides, peut-être en raison de l'existence d'une hydrolyse d'une fraction mineure des TG (lipolyse) (tableau 1).

\section{Composition en AG à chaîne longue}

L'apport alimentaire d'AG insaturés par la graine de lin ( $L$ ) ou le mélange de graines de colza plus lin $(\mathrm{CL})$ augmente significativement les proportions de 18:3n-3 (+56 et $+36 \%$ respectivement), des 18:1 trans totaux (+66 et $+105 \%)$ et du 9cis, 11 trans 18:2 (CLA) (+50 et $+41 \%$ ) dans le muscle LT (tableau 2), le 18:3 $\mathrm{n}$-3 et le CLA étant connus pour être bénéfiques pour la santé humaine.

De telles augmentations s'effectuent au détriment des AG cis mono-insaturés principalement et non pas des AG saturés. Les mêmes effets sont également observés avec le muscle ST, avec des teneurs plus élevées en 18:3 n-3 (+25\%) et en CLA (+27\%) que le muscle LT. Des effets similaires significatifs concernent les 
AGPI n-3 entraînant une baisse du rapport AGPI n-6/AGPI n-3 (3,3 vs 4,5) plus bénéfique à I'Homme (tableau 2).

Ces résultats confirment des observations antérieures sur muscles LT et Rectus abdominis de taurillons recevant le même supplément en graines de lin extrudées, avec une augmentation parallèle de cis, 11 trans 18:2 (CLA) et son précurseur le 18:1 $\Delta 11$ trans (acide vaccénique) (Bauchart et al., 2005), bénéfiques pour I'Homme par son action hypocholestérolémique (Bauchart et al., 2007).

En revanche, de telles augmentations observées chez nos bovins relativement gras et âgés sont, en intensité, plus faibles que celles observées chez des sujets plus jeunes et plus maigres. Cela suggère une baisse de la réactivité des muscles (et de leurs tissus gras associés) avec l'âge et le dépôt de gras vis-à-vis de l'apport d'AGPI alimentaires.

L'analyse CPG séparée des AG des fractions polaire (dominée par les PL) et neutre (dominée par les TG), purifiées par chromatographie liquide sur cartouche de silice activée par des groupements aminopropyles, montre clairement des compositions spécifiques en AG des deux familles de lipides (Bauchart et al., 2005). Ainsi, la fraction polaire est environ dix fois plus riche en AGPI (25 vs 2,5 \%) que la fraction neutre, laquelle est dominée par les AG saturés et mono-insaturés (données non fournies). La composition en AG de ces fractions lipidiques est modifiée par les suppléments lipidiques, notamment les AGPI n-3 des PL du muscle ST des vaches recevant le régime à base de graines de lin (+55 \%) (Habeanu et al., 2008b).

Tableau 1. Effets des suppléments lipidiques alimentaires sur les teneurs ( $g / 100 \mathrm{~g}$ de tissu frais) en différentes classes de lipides du muscle Longissimus thoracis (LT) chez les vaches de réforme normandes en finition (Habeanu et al., 2008a ; Habeanu et al. 2008b).

\begin{tabular}{|lccccc|}
\hline Régimes & T & L & CL & SEM & $\boldsymbol{p}$ \\
\hline Matière sèche (\%) & 26,93 & 26,73 & 27,13 & 0,42 & 0,81 \\
\hline Lipides totaux & 4,70 & 3,29 & 3,46 & 0,46 & 0,79 \\
\hline Triglycérides & 3,79 & 3,03 & 2,87 & 0,41 & 0,69 \\
\hline Phospholipides & 0,70 & 0,68 & 0,69 & 0,01 & 0,61 \\
\hline Esters de cholestérol & $0,03^{\text {a }}$ & $0,05^{\text {b }}$ & $0,01^{\text {a }}$ & 0,04 & 0,03 \\
\hline Cholestérol libre & 0,07 & 0,06 & 0,06 & 0,005 & 0,52 \\
\hline Diglycérides & 0,05 & 0,07 & 0,05 & 0,02 & 0,38 \\
\hline Acides gras libres & $0,06^{\mathbf{a}}$ & $0,19^{\mathbf{b}}$ & $0,05^{\mathbf{a}}$ & 0,03 & 0,03 \\
\hline
\end{tabular}

$\mathrm{a}, \mathrm{b}, \mathrm{c} p \leq 0,10 ; \mathrm{L}=\operatorname{lin} ; \mathrm{CL}=\operatorname{lin}+$ colza.

Tableau 2. Effets des suppléments lipidiques sur la composition en acides gras des lipides totaux du muscle Longissimus thoracis des vaches de réforme en finition (Habeanu et al., 2008b).

\begin{tabular}{|c|c|c|c|c|c|}
\hline Acides gras & C & $\mathbf{L}$ & $\mathbf{C L}$ & SEM & $\boldsymbol{p}$ \\
\hline \multicolumn{6}{|l|}{$\%$ Acides gras totaux } \\
\hline $18: 3 n-3$ & $0,39^{\mathrm{A}}$ & $0,61^{\mathrm{B}}$ & 0,53 & 0,05 & 0,05 \\
\hline cis9, trans11-18-2 (CLA) & 0,32 & 0,48 & 0,45 & 0,05 & 0,08 \\
\hline$\Sigma$ AG saturés & 47,01 & 48,22 & 48,29 & 0,80 & 0,44 \\
\hline$\Sigma$ AGMI cis & $45,38^{A}$ & $41,56^{\mathrm{B}}$ & $40,90^{\mathrm{B}}$ & 0,59 & $<0,0001$ \\
\hline$\Sigma$ AGMI trans & $2,46^{A}$ & $4,08^{\mathrm{Bb}}$ & $5,04^{\mathrm{Cc}}$ & 0,32 & $<0,0001$ \\
\hline$\Sigma$ AGPI n-3 & 0,90 & 1,23 & 1,02 & 0,11 & 0,17 \\
\hline$\Sigma$ AGPI n-6 & 4,03 & 4,05 & 3,80 & 0,26 & 0,77 \\
\hline AGPI n-6/AGPI n-3 & $4,54^{\mathrm{A}}$ & $3,32^{\mathrm{B}}$ & 4,10 & 0,28 & 0,028 \\
\hline AGPI/AG saturés & 0,11 & 0,12 & 0,11 & 0,009 & 0,80 \\
\hline AG totaux ( $\mathrm{g} / 100 \mathrm{~g}$ de tissu frais) & 3,95 & 3,64 & 3,63 & 0,41 & 0,64 \\
\hline
\end{tabular}

$a, b, c p \leq 0,05 ;{ }^{A, B, C} p \leq 0,01$.
L'addition d'antioxydants (vit E + EVRP) renforce l'effet stimulant des suppléments lipidiques sur les trois AG considérés (18:3 n-3, total trans 18:1, 9cis, 11 trans 18:2) dans les lipides totaux des deux muscles LT et ST (données non fournies). Les mêmes effets des antioxydants, déjà observés chez le jeune taurillon charolais recevant des suppléments en graines de lin extrudées, suggèrent l'existence d'une modulation, par les antioxydants alimentaires (vit E), du métabolisme microbien des AG dans le rumen (Bauchart et al., 2005).

\section{Isomères trans du 18:1}

L'analyse par CPG-spectrométrie de masse des différents isomères trans du 18:1 (purifiés par HPLC préparative) montre clairement qu'avec la ration de base riche en aliment concentré (à base de céréales), les suppléments lipidiques peuvent diversement modifier la valeur santé des isomères trans du 18:1 de la viande sur la base des isomères $\Delta 9 \operatorname{tr}$ et $\Delta 10 \operatorname{tr}$ (indésirables) et $\Delta 11$ tr 18:1 (bénéfique) (tableau 3). Les suppléments lipidiques exercent un effet positif lorsque le 18:3 n-3 est principalement apporté (régime L) ou plutôt négatif quand le 18:3 n-3 est associé au 18:1 n-9 (régime CL) (Bispo Villar et al., 2009).

\section{Effets des facteurs alimentaires et physiologiques sur la lipoperoxydation dans le plasma et la viande chez la vache de réforme}

La viande bovine de meilleure valeur nutritionnelle (teneur plus élevée en AGPI n-3), associée à une meilleure protection des AGPI par les antioxydants par la mise en œuvre de facteurs d'élevage, est commercialisée avec une haute valeur ajoutée sous I'appellation, par exemple, de Label rouge. Dans ce contexte, il est nécessaire de préciser l'impact des traitements technologiques spécifiquement appliqués à la viande pour préserver leurs qualités jusqu'à leur commercialisation. En fait, les traitements de maturation et surtout de conditionnement des viandes peuvent stimuler les voies de peroxydation et de lipolysis qui impactent très négativement les caractéristiques de santé (accumulation de lipoperoxydes toxiques) et les propriétés sensorielles (couleur, goût) des viandes (Min et Ahn, 2005).

Dans ce contexte, les AGPI des PL sont les substrats primaires de la peroxydation lipidique (lipoperoxydation) des produits carnés, alors que les TG seraient peu impliqués dans le processus. L'incorporation des AGPI (fournis par des graines oléagineuses ou de l'herbe jeune) dans les lipides musculaires du bovin n'active 
Tableau 3. Effets des suppléments lipidiques ( $L=$ lin ; $C=$ colza) sur la distribution des isomères trans du 18:1 (en pourcentage des trans 18:1 totaux, moyenne $\pm E T$ ) des lipides totaux du muscle LT bovin (Bispo Villar et al., 2009).

\begin{tabular}{|c|c|c|c|c|c|c|c|c|c|c|c|}
\hline $\begin{array}{l}\text { Trans } \\
18: 1\end{array}$ & 6 tr & $7 \operatorname{tr}$ & 8 tr & $9 \mathrm{tr}$ & 10 tr & 11 tr & $12 \operatorname{tr}$ & $13 t r$ & 14tr & 15 tr & $16 \mathrm{tr}$ \\
\hline Régime T & $\pm 0,9$ & $0,5 \pm 0,1$ & $1,9 \pm 0,3$ & $5 \pm 1,5$ & $3,7 \pm 18,6$ & $5,1 \pm 14,4$ & $4,3 \pm 1,2$ & $3,4 \pm 1,0$ & $4,0 \pm 1,3$ & $3,4 \pm 1,8$ & $2,9 \pm 1,9$ \\
\hline Régime L & $0,6 \pm 0,5$ & $0,4 \pm 0,1$ & $1,6 \pm 0,4$ & $5,0^{*} \pm 0,8$ & $15,6^{*} \pm 6,7$ & $33,2 \pm 11,8$ & $6,1^{*} \pm 0,3$ & $8,7^{*} \pm 0,8$ & $9,1^{*} \pm 0,9$ & $10,9^{*} \pm 9,0$ & $8,9^{*} \pm 2,7$ \\
\hline égime $\mathrm{CL}$ & $0,5 \pm 0,4$ & $0,6 \pm 0,1$ & $2,3 \pm 0,6$ & $6,4^{*} \pm 0,9$ & $41,1 \pm 16,4$ & $25,0^{*} \pm 12,4$ & $4,9 \pm 9,2$ & $5,8 \pm 1,8$ & $5,5 \pm 1,6$ & $5,0 \pm 1,8$ & $3,1 \pm 1,1$ \\
\hline
\end{tabular}

${ }^{*} p<0,05$.

pas les enzymes antioxydantes du muscle, la protection vis-à-vis du processus de lipoperoxydation étant strictement dépendante de la quantité et de l'activité des molécules antioxydantes présentes dans les cellules musculaires (Durand et al., 2005).

Dans cette étude, les effets des traitements technologiques sur la stabilité des lipides musculaires à la peroxydation ont été étudiés sur des viandes issues de muscles de vaches ayant reçu le régime lin riche en AGPI n-3 (AG parmi les plus sensibles à la peroxydation), enrichi ou non en antioxydants (vit E \pm EVRP). L'impact des conditions de maturation et de conditionnement des viandes a été plus particulièrement étudié (collaboration avec ADIV, ClermontFerrand).

\section{Aspects expérimentaux}

\section{Animaux et régimes}

L'étude a été réalisée sur des viandes issues d'animaux ayant reçu les mêmes régimes (ration à base de concentré et de paille, suppléments lipidiques et en antioxydants), dans les mêmes conditions de stress des animaux (le stress émotionnel favorise les réactions de lipoperoxydation sanguine et tissulaire) que celles de l'étude des lipides et des AG de la viande (cf. «Partie I : Effets des AGPI n-3 et des antioxydants alimentaires sur les $A G$ de la viande bovine »). L'intensité de stress émotionnel des animaux a été déterminée par les mesures de la fréquence cardiaque et du taux de cortisol plasmatique :

- à la fin de la période de finition ;

- juste après l'abattage au cours de l'égorgement des animaux.

\section{Traitements technologiques des viandes}

Les muscles LT et ST ont été collectés juste après l'abattage, puis conservés à $+4{ }^{\circ} \mathrm{C}$ sous vide pendant 12 jours et finalement découpés sous forme de steaks comme dans la pratique. Les steaks ont été conditionnés à $+4{ }^{\circ} \mathrm{C}$ en barquette recouverte d'un film :

- sous air pendant quatre jours ;

- sous atmosphère modifiée $\left(70: 30, \mathrm{O}_{2} / \mathrm{CO}_{2}\right)$ pendant sept jours ;

- en sac placé sous vide pendant 14 jours.
Les échantillons de viande sont ensuite broyés en poudre dans l'azote liquide et stockés à $-80^{\circ} \mathrm{C}$ jusqu'aux mesures de lipoperoxydation.

\section{Méthodes de caractérisation de la lipoperoxydation}

L'intensité de lipoperoxydation dans le plasma (veine jugulaire) et les échantillons de viande à l'abattage a été déterminée par la mesure du taux de production du malonedialdéhyde (MDA) extrait par I'hexane, puis analysé par HPLC (détection en fluorescence) avec une courbe de calibration utilisant le tétraéthoxypropane. La concentration en vitamine $\mathrm{E} d u$ plasma et des muscles a été déterminée par HPLC et détection UV.

La susceptibilité in vitro de lipoperoxydation du plasma a été évaluée par :

- la mesure de la cinétique de production des diènes conjugués (DC) indiquant le degré de résistance du milieu biologique à la lipoperoxydation (phase de résistance ou lag phase) ;

- la vitesse de la réaction de lipoperoxydation (Tx max) ;

- la quantité maximale de DC produits (Q $\max$ ).

L'index de peroxydabilité (IP) des AG de la viande a été calculé à partir de la composition en $A G$ des lipides totaux de la viande selon l'équation : $\mathbb{P}=($ pourcentage diènoique $\times 1)$ + (pourcentage triènoique $\times 2)+($ pourcentage tétraènoique $\times 3)+($ pourcentage pentaènoique $\times 4)+($ pourcentage hexaènoique $\times 5$ ). Cet index détermine le nombre $d^{\prime}$ atomes d'hydrogène bis-allyliques des AGPI et donc leur susceptibilité relative à la peroxydation. Enfin, les hydroxyalkénals libres plasmatiques de type 4-hydroxy-2-nonènal (4$\mathrm{HNE}$ ) et 4-hydroxy-2-hexènal (4-HHE), des marqueurs spécifiques de la peroxydation des AGPI $n-6$ et $n-3$ respectivement, ont été déterminés par CPG couplée à la spectrométrie de masse.

\section{Résultats}

\section{Effets des n-3 PUFA et des antioxydants alimentaires sur la lipoperoxydation plasmatique et tissulaire}

\section{Lipoperoxydation du plasma}

Les suppléments lipidiques de la ration riches en AGPI n-3 réduisent la capacité du plasma à résister à la lipoperoxydation (-11\%), favorisant la formation des DC $(\times 1,75)$ et du MDA (× 2) (tableau 4).

Seul, I'apport combiné d'antioxydants (vit E + EVRP) protège efficacement les lipides plasmatiques de la peroxidation (régime LEP) favorisée par les suppléments alimentaires en AGPI $\mathrm{n}-3$, la teneur en MDA et la vitesse de lipoperoxydation Tx étant diminuées de -80 et $-33 \%$ $(p=0,01)$ respectivement avec le régime LEP comparé au régime $L$ (tableau 4 ).

Une telle protection par le mélange vit E + EVRP (régime LEP) agirait non seulement au cours de la phase de propagation de la réaction de lipoperoxydation (la vit E agirait comme casseur de chaînes), mais aussi dès la phase d'initiation de la réaction par la fixation des radicaux libres par les polyphénols végétaux (EVRP), comme cela a

Tableau 4. Effets des AGPI n-3 et des antioxydants alimentaires sur les marqueurs plasmatiques de la lipoperoxydation chez la vache de réforme en finition (Gobert et al., 2009a).

\begin{tabular}{|llllll|}
\hline Régimes & T & L & LE & LEP & Prob \\
\hline MDA $(\mu \mathrm{g} / \mathrm{mL})$ & $0,05 \pm 0,02^{\mathrm{a}}$ & $0,10 \pm 0,06^{\mathrm{b}}$ & $0,05 \pm 0,04^{\mathrm{ab}}$ & $0,02 \pm 0,02^{\mathrm{a}}$ & $* *$ \\
\hline$\alpha$-tocophérol $(\mu \mathrm{g} / \mathrm{mL})$ & $1,82 \pm 0,80^{\mathrm{a}}$ & $4,11 \pm 1,15^{\mathrm{b}}$ & $9,65 \pm 5,65^{\mathrm{c}}$ & $11,05 \pm 5,36^{\mathrm{d}}$ & $* * *$ \\
\hline Lag phase $(\min )$ & $13,2 \pm 0,8^{\mathrm{a}}$ & $11,7 \pm 0,8^{\mathrm{b}}$ & $11,8 \pm 2,0^{\mathrm{ab}}$ & $15,2 \pm 4,3^{\mathrm{ab}}$ & $* *$ \\
\hline Tx max $\left(\mathrm{A}_{234} / \mathrm{min}\right)$ & $8,04 \pm 2,51^{\mathrm{ab}}$ & $9,52 \pm 0,12^{\mathrm{a}}$ & $8,89 \pm 2,58^{\mathrm{ab}}$ & $6,32 \pm 1,38^{\mathrm{b}}$ & $* * *$ \\
\hline Q max $(\mathrm{DC} \max )$ & $217 \pm 58^{\mathrm{a}}$ & $379 \pm 27^{\mathrm{b}}$ & $347 \pm 115^{\mathrm{b}}$ & $375 \pm 52^{\mathrm{b}}$ & $*$ \\
\hline
\end{tabular}

${ }^{*} p<0,1 ;{ }^{* *} p<0,05 ;{ }^{* * *} p<0,01$.

$\mathrm{a}, \mathrm{b}, \mathrm{c}, \mathrm{d} p<0,05$. 
été déjà montré chez le rat et le mouton (Gladine et al., 2007) ainsi que chez la vache en lactation (Gobert et al., 2009a). Chez la vache de réforme en finition soumise à un stress émotionnel deux heures avant l'abattage, le niveau de lipoperoxydation du plasma évalué par le niveau de MDA (tableau 5) n'était pas augmenté dans les lots d'animaux stressés comparés à ceux des lots non stressés. Cependant, des marqueurs spécifiques de l'oxydation des AGPI $\mathrm{n}-3$ et n-6 (4-HNE et 4-HHE respectivement) (tableau 5) tendaient à baisser chez les vaches recevant la vit E et les EVRP (lot LEP) ( $p=0,07$ et NS, respectivement).

\section{Lipoperoxidation de la viande bovine}

\section{Effets des traitements technologiques}

À l'abattage (j0), la teneur en MDA du muscle LT est comparable chez les vaches recevant le régime témoin $(T)$ et le même régime supplémenté en graines de lin seules $(\mathrm{L})$ ou associées au mélange d'antioxydants constitués de vit $E$ et des EVRP (LEP), la valeur de l'index de peroxyda- tion des muscles étant comparable pour les trois régimes (tableau 6). En revanche, après maturation sous vide pendant 12 jours (j12), la mise au contact des tranches de viande au cours du conditionnement en barquette avec $\mathrm{I}^{\prime} \mathrm{O}_{2}$ de I'air (viande sous film perméable à l'air) ou $d^{\prime}$ une atmosphère modifiée $\left(70 \% d^{\prime} \mathrm{O}_{2} / 30 \%\right.$ de $\mathrm{CO}_{2}$ ) stimule la lipoperoxydation de la viande (tableau 6). Les effets les plus marqués sont observés pour les viandes issues des vaches ayant reçu le supplément en graines de lin mises au contact de l'air $(\times 10,4 ; p<0,05)$ et surtout de $70 \% d^{\prime} \mathrm{O}_{2}(\times 21,8 ; p<0,05)$ par rapport à la viande conditionnée sous vide (tableau 6) (Gobert et al., 2008, 2010).

Même pour des valeurs de PI similaires des viandes, l'intensité de lipoperoxydation sous atmosphère modifiée tend à être plus élevée avec le lot lin $(2,96 \mu \mathrm{g}$ de $\mathrm{MDA} / \mathrm{g}$ tissu) que le lot témoin $(2,19 \mu \mathrm{g}$ de MDA/g de tissu) $[p=0,1]$. Cela pourrait être le résultat d'une plus grande sensibilité des viandes à I'oxydation des AGPI n-

Tableau 5. Effets des traitements alimentaires (L vs LEP) et des conditions de stress émotionnel préabattage (sans stress vs avec stress) sur l'intensité des indicateurs d'intensité de lipoperoxydation et du statut en antioxydant du plasma des vaches de réforme prélevé juste après l'abattage (Gobert et al., 2009b).

\begin{tabular}{|c|c|c|c|c|c|c|c|}
\hline \multirow{2}{*}{\begin{tabular}{|l|} 
Régimes \\
Traitements
\end{tabular}} & \multicolumn{2}{|l|}{$\mathbf{L}$} & \multicolumn{2}{|l|}{ LEP } & \multicolumn{3}{|c|}{ Valeurs de $p$ - } \\
\hline & $\begin{array}{l}\text { Sans } \\
\text { stress }\end{array}$ & $\begin{array}{l}\text { Avec } \\
\text { stress }\end{array}$ & $\begin{array}{l}\text { Sans } \\
\text { stress }\end{array}$ & $\begin{array}{l}\text { Avec } \\
\text { stress }\end{array}$ & Antioxydant & Stress & Antioxydant $\times$ Stress \\
\hline $\operatorname{MDA}(\mu \mathrm{g} / \mathrm{mL})$ & 0,06 & 0,07 & 0,06 & 0,05 & NS & NS & NS \\
\hline HNE (ng/mL) & 3,5 & 2,5 & 1,9 & 2,1 & 0,07 & NS & NS \\
\hline HHE (ng/mL) & 0,45 & 0,42 & 0,38 & 0,34 & NS & NS & NS \\
\hline Vit $E(\mu \mathrm{g} / \mathrm{mL})$ & 3,17 & 2,91 & 7,75 & 8,77 & $<0,0001$ & NS & NS \\
\hline
\end{tabular}

Tableau 6. Concentration en malondialdehyde (MDA) [ $\mu \mathrm{g} / \mathrm{g}$ de tissu frais] du muscle Semitendinosus des vaches recevant le régime témoin $(T)$ ou le même régime de base enrichi en lin $(L)$ ou en lin + antioxydants (LEP). Le MDA a été dosé dans les viandes à l'abattage (j0), après maturation sous vide (j12) suivie d'un conditionnement de 14 jours sous vide, de sept jours sous film perméable à l'air ou de quatre jours sous atmosphère modifiée (70:30, $\mathrm{O}_{2} / \mathrm{CO}_{2}$ ) (Gobert et al., 2010).

\begin{tabular}{|c|c|c|c|c|c|}
\hline \multirow[b]{3}{*}{$\begin{array}{l}\text { MDA } \\
\text { ( } \mu \mathrm{g} / \mathrm{g} \text { de tissu frais) }\end{array}$} & \multicolumn{5}{|c|}{ Modes de traitement des viandes } \\
\hline & \multicolumn{2}{|c|}{ Maturation sous vide } & \multicolumn{3}{|c|}{ Conditionnement en barquette } \\
\hline & à j0 & à j12 & Sous vide & $\begin{array}{l}\text { Sous film } \\
\text { perméable }\end{array}$ & $\begin{array}{l}\text { Sous atmosphère } \\
\text { modifiée }\end{array}$ \\
\hline $\mathrm{T}$ & $0,16^{\mathrm{a}} \pm 0,05$ & $0,15^{\mathrm{a}} \pm 0,01$ & $0,16^{\mathrm{ab}} \pm 0,04$ & $1,10^{b} \pm 0,38$ & $2,19^{c} \pm 1,88$ \\
\hline $\mathrm{L}$ & $0,14^{\mathrm{a}} \pm 0,04$ & $0,19^{\mathrm{a}} \pm 0,09$ & $0,19^{a} \pm 0,09$ & $1,41^{b} \pm 0,72$ & $2,96^{c} \pm 1,36$ \\
\hline LEP & $0,16^{\mathrm{a}} \pm 0,05$ & $0,14^{\mathrm{a}} \pm 0,03$ & $0,14^{a} \pm 0,02$ & $0,65^{\mathrm{a}} \pm 0,49$ & $0,93^{\mathrm{a}} \pm 0,82$ \\
\hline \multicolumn{6}{|l|}{ Diet effect } \\
\hline T vs L & 0,9 & 0,9 & 0,9 & 0,5 & 0,1 \\
\hline T vs LEP & 0,6 & 0,7 & 0,8 & 0,3 & 0,01 \\
\hline L vs LEP & 0,6 & 0,8 & 0,8 & 0,09 & $<0,0001$ \\
\hline
\end{tabular}

$a, b, c p<0,05$.
3 (lot L), comme cela a été rapporté chez le bouvillon recevant des suppléments lipidiques riches en AGPI (Campo et al., 2006).

\section{Effet des antioxydants alimentaires}

L'apport alimentaire conjoint, pendant 100 jours, de vit $E$ et des EVRP protège efficacement la viande de la lipoperoxydation puisque les teneurs en MDA sont toujours inférieures à $1 \mu \mathrm{g} / \mathrm{g}$ de tissu considéré comme la valeur limite de tolérance à la flaveur rance de la viande du consommateur (Campo et al., 2006). De même, ce mélange en antioxydants protège les AGPI de la viande de certaines conditions très pro-oxydantes de conditionnement, telles que le contact des viandes tranchées avec $\mathrm{I}^{\prime} \mathrm{O}_{2}$ de l'air en conditionnement sous film perméable à l'air $(p<0,09)$ et surtout sous atmosphère modifiée (70 $\%$ en $\mathrm{O}_{2}$, $p<0,0001$ ) (tableau 6) (Gobert et al., 2010).

Comme décrit précédemment (Gatellier et al., 2000), la vit E apportée seule n'est pas assez efficace pour protéger la viande de la lipoperoxydation chez des bovins recevant des suppléments en AGPI n-3. En revanche, comme chez le rat et le mouton (Gladine et al., 2007), et la vache laitière en lactation (Gobert et al., 2009a), I'association de la vit $\mathrm{E}$ avec les EVRP exercerait des actions synergiques résultant de leurs propriétés respectives lipophiles (vit E) et hydrophiles (polyphénols). La vit E agirait comme un antioxydant casseur de chaînes, et les polyphénols comme piégeurs de radicaux libres. Cela expliquerait ainsi l'efficacité de leur association face à la lipoperoxydation de la viande, même dans les conditions de stockage favorisant fortement les processus de lipoperoxydation.

\section{Effets des AGPI n-3 et des antioxydants alimentaires sur la couleur des viandes}

L'objectif de l'étude était d'analyser sur les viandes bovines, après maturation et conditionnement, le possible effet négatif de la supplémentation en AGPI n-3 de la ration :

- sur la couleur de la viande chez des bovins soumis à un stress émotionnel deux heures avant l'abattage ;

- de déterminer le possible effet protecteur des antioxydants apportés pendant 100 jours au cours de la phase de finition.

La mesure des caractéristiques de couleur a été réalisée par spectrophotométrie avec une sphère $d$ 'intégration et à l'aide du système CIELAB. À partir du spectre de réflectance dans le visible (360 à $760 \mathrm{~nm}$ ), les données ont concerné la luminosité $\left(L^{*}\right)$ et les intensités de rouge $\left(a^{*}\right)$ et de jaune $\left(b^{*}\right)$. Les différences de réflectance ont été utilisées pour le calcul de l'index d'oxygénation (R630-R580 ; oxygénation de la myoglobine) et le pourcentage de myoglobine (M) sous forme de metmyoglobine $(\mathrm{MB})$ responsable de la couleur brune de la viande. 
Les résultats montrent que lorsque la viande est conditionnée sous vide, les valeurs des paramètres de couleur ne sont pas influencées par les conditions alimentaires ni par le niveau émotionnel des animaux. En revanche, conditionnées sous film perméable à l'air, les viandes des vaches du lot lin ( $L$ ) sont plus rouges que celles du lot témoin. Dans le cas des animaux soumis à un stress émotionnel, l'apport des antioxydants vit $\mathrm{E}+$ EVRP aux animaux du lot lin maintient seul la couleur rouge des viandes conditionnées sous film perméable à l'air. Sous atmosphère modifiée riche en $\mathrm{O}_{2}$, favorisant l'oxygénation de la myoglobine, la viande est plus rouge que celle conditionnée sous film (résultats non illustrés), alors que l'intensité de rouge est plus faible pour les viandes issues d'animaux stressés recevant le supplément lipidique à base de lin sans antioxydants (figure 1).

En fait, les vaches stressées recevant du lin sans antioxydants sont beaucoup plus sensibles à la peroxydation en relation avec la perte de couleur rouge consécutive à un taux de metmyoglobine plus élevé $(p<0,0001)$. Cependant, quels que soient les paramètres de couleur mesurés (intensité de rouge, index d'oxygénation, pourcentages de $\mathrm{M}$ et $\mathrm{MB}$ ), la supplémentation en antioxydants (vit E + EVRP) de la ration lin entraîne des caractéristiques de couleur similaires de celles des viandes des animaux du lot témoin sans ajout de lipides et d'antioxydants. Elle permettrait, en outre, d'inhiber le phénomène d'oxydation généré par le stress émotionnel des animaux (figure 1). Cette étude montre clairement que l'apport de vit E dans la ration améliore l'intensité de couleur de la viande conditionnée sous atmosphère modifiée riche en $\mathrm{O}_{2}$. De plus, elle montre que la couleur de la viande peut être nettement dégradée par le stress émotionnel des animaux recevant une ration enrichie en graines de lin dont les AGPI n-3 sont très sensibles à la peroxydation. Dans ces conditions, I'apport alimentaire conjoint de vit E et de polyphénols (EVRP) représente un moyen très efficace de prévenir à la fois l'altération de la couleur et le développe- ment de la lipoperoxydation des viandes chez le bovin en finition.

\section{Conclusion}

Chez les bovins producteurs de viande en période de finition, l'apport de graines de lin extrudées riches en AGPI n-3 améliore la valeur nutritionnelle de la viande en favorisant I'incorporation du 18:3 n-3 et sa conversion en AGPI LC n-3 (notamment EPA et DPA) dans les lipides des muscles, particulièrement dans les $P L$ des membranes cellulaires. De plus, une telle stratégie nutritionnelle favoriserait également le dépôt d'acide linoléique conjugué (CLA), spécialement l'acide ruménique (9cis, 11 trans 18:2) et son précurseur l'acide vaccénique (18:1 11 trans), tous deux connus pour être bénéfiques pour la santé humaine par leur propriété anticholestérolémique. En revanche, l'apport conjoint de graines de colza (deux tiers) riches en 18:1 $\Delta 9$ cis et de graines de lin (un tiers) riches en 18:3 n-3 limiterait (à quantité comparable d'AG apportés dans la ration) le dépôt musculaire de 18:3 n-3 et favoriserait le dépôt des isomères trans du 18:1 de type 18:1 $\Delta$ trans et surtout 18:1 $\Delta 10$ trans, connus pour être toxiques pour l'Homme.

L'absorption accrue d'AGPI n-3 par les bovins avec le régime lin stimulerait la lipoperoxydation :

- dans son organisme entier, entraînant un risque pour le bon fonctionnement des tissus et des organes (stress oxydant) et pour la santé et les performances des animaux ;

- sur les tissus musculaires, avec le risque d'accumulation de produits oxydés (MDA, 4$\mathrm{HHE}, 4-\mathrm{HNE}$ ) altérant la valeur santé des viandes pour le consommateur.

Pour éviter de tels risques préjudiciables à l'animal et au consommateur, l'emploi d'antioxydants dans les rations des bovins est fortement recommandable. Ce travail a montré que la vit $\mathrm{E}$ alimentaire exerce un effet protecteur plus faible vis-à-vis de la lipoperoxydation que le mélange vit E + EVRP riche en polyphénols. Une stratégie alimentaire combinant l'emploi

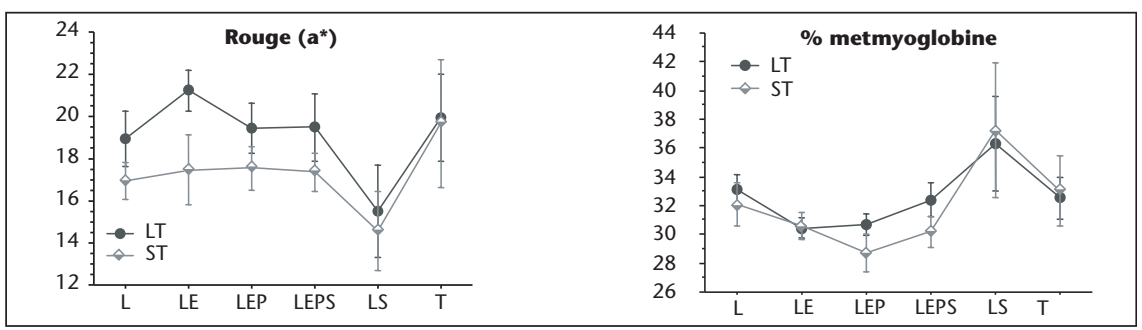

Figure 1. Effets des suppléments en antioxydants (E, EP) et du stress émotionnel $(S)$ des vaches recevant la ration témoin $(T)$ ou enrichie en lin $(L)$ sur les paramètres de couleur des viandes issues des muscles $L T$ et ST conditionnées sous atmosphère modifiée (Parafita-Thomas et al., 2008). de suppléments lipidiques riches en AGPI n-3 et du mélange en antioxydants (vit E + polyphénols) éviterait les risques majeurs de lipoperoxydation des viandes quels que soient :

- les conditions de stockage des viandes (maturation, conditionnement notamment en présence $d^{\prime} \mathrm{O}_{2}$ ) ;

- le niveau de stress émotionnel des animaux juste à l'approche de l'abattage.

Enfin, cette stratégie alimentaire permettrait d'éviter tout risque d'altération de la couleur des viandes (notamment celles des animaux stressés) en parallèle aux processus de lipoperoxydation. Elle participerait ainsi à une grande stabilité des viandes, bénéfiques notamment pour les activités des industries de transformation des viandes et de la grande distribution.

\section{RÉFÉRENCES}

Afssa. Apports nutritionnels conseillés pour la population française. Tec et Doc Éditions, $3^{\text {ème }}$ édition 2001.

Bauchart D, Gladine C, Gruffat D, et al. Effects of diets supplemented with oils and vitamin $E$ on specific fatty acids of rectus abdominis muscle in charolais fattening bulls. In: Indicators of milk and beef quality, EAAP Publ, $n^{\circ} 112$, Hocquette JF, Gigli S (eds) Wageningen Acad, Publishers, 2005, pp 431-6.

Bauchart D, Roy A, Lorenz $S$, et al. Dietary supply of butter rich in trans 18:1 isomers or in 9cis, 11trans conjugated linoleic acid affects plasma lipoproteins in hypercholesterolemic rabbits. Lipids 2007 ; 42 : 123-33.

Bispo Villar E, Thomas A, Lyan B, et al. Lipid supplements rich in $n-3$ polyunsaturated fatty acids deeply modify trans 18:1 isomers in the Longissimus thoracis muscle of finishing bovine. In the proceedings of the 11 th International Symposium on Ruminant Physiology, 6-9th September 2009, Clermont-Ferrand, France, 2009, pp 464-6.

Campo MM, Nute GR, Hughes SI, et al. Flavour perception of oxidation in beef. Meat Sci 2006; 72 : 303-11.

Durand D, Scislowski V, Chilliard Y, et al. High fat rations and lipid peroxidation in ruminants; consequences on animal health and quality of products. In: Indicators of milk and beef quality, EAAP Publ, $\mathrm{n}^{\circ} 112$. Hocquette JF, Gigli $S$ (eds), Wageningen Acad, Publishers, 2005, pp 137-50.

Folch J, Lees M, Sloane-Stanley GHS. A simple method for the isolation and purification of total lipids from animal tissues. J Biol Chem 1957; 226 : 497-509.

Gatellier P, Mercier Y, Rock E, et al. Influence of dietary fat and vitamin $E$ supplementation on free radical production and on lipid and protein oxidation in turkey muscle extracts. J Agric Food Chem 2000 ; 48 : 1427-33. 
Geay Y, Bauchart D, Hocquette JF, et al. Effect of nutritional factors on biochemical, structural and metabolic characteristics of muscles in ruminants; consequences on dietetic value and sensorial qualities of meat. Reprod Nutr Dev 2001 ; 41 : 1-26.

Gladine C, Morand C, Rock E, et al. Plant extracts rich in polyphenols (PERP) are efficient antioxidants to prevent lipoperoxidation in plasma lipids from animals fed n-3 PUFA supplemented diets. Anim Feed Sci Technol 2007 ; 136 : 281-96.

Gobert M, Bauchart D, Parafita E, et al. Dietary vitamin $E$ associated with plant polyphenols effectively protect from lipoperoxidation in processed meats in the finishing bovine given an n-3 PUFA-rich diet. In the Proceedings of the 54th International Congress of Meat Science and Technology, 10-15th August 2008, Cape Town, South Africa, 3A4, 2008.

Gobert M, Bauchart D, Parafita E, et al. Plant extracts combined with vitamin $\mathrm{E}$ in PUFA-rich diets for finishing cattle protect processed beef against lipoperoxidation. Meat Science 2010 (accepted).

Gobert M, Martin B, Ferlay A, et al. Plant polyphenols associated with vitamin $E$ are susceptible to reduce plasma lipoperoxidation in dairy cows given n-3 polyunsaturated fatty acids. J Dairy Sci 2009a ; 92 : 6095-104.
Gobert M, Bourguet C, Terlouw C, et al. Preslaughter stress and lipoperoxidation: protective effect of vitamin $E$ and plant extracts rich in polyphenols given to finishing cattle. In the Proceedings of the 11th International Symposium on Ruminant Physiology, 6-9th September 2009, France: ClermontFerrand, 2009b, pp 814-5.

Habeanu M, Durand D, Gobert M, Bauchart D. Lipids and fatty acid composition of Longissimus thoracis and Semitendinosus muscles in finishing Normand cows. Archiva Zootechnica 2008b ; 11 : 21-9.

Habeanu M, Durand D, Gobert M, et al. Lipid and fatty acid composition of Longissimus thoracis and Semitendinosus muscles in finishing Normand cows (fat breed) given unsaturated lipid supplements. In the Proceedings of the 54th Intern. Cong Meat Sci Technol 10-15th August 2008, Cape Town, South Africa, 2B5, 2008a.

Min B, Ahn DU. Mechanisms of lipid peroxidation in meat and meat products. A review. Food Sci Biotechnol $2005 ; 14$ : 152-63.

Narayan B, Miyashita K, Hosakawa M. Physiological effects of eicosapentaenoic acid (EPA) and docosahexaenoic acid (DHA) - a review. Food Rev Intern 2006 ; $22: 291-307$.
Normand J, Bastien D, Bauchart D, et al. Production of beef enriched in n-3 PUFA by given linseeds to the animals: different ways for providing linseeds in diets and consequences on beef quality? Renc Rech Ruminants $2005 ; 12$ : 359-66.

Parafita-Thomas E, Vialter S, Gobert M, et al. Dietary supply of antioxidants to finishing cattle can improve the stability of meat colour, even in animals stressed just before slaughter. In: 13th meeting of muscle sciences and meat technology (JSMTV), France: Tours, 2008, pp 91-2.

Reynolds H, Anderson MK. Rapid separation and quantitation of combined neutral and polar lipid classes by high-performance liquid chromatography and evaporative light-scattering mass detection. / Chromat $B 1998 ; 708: 21-6$.

Scollan N, Richardson I, De Smet S, et al. Enhancing the content of beneficial fatty acids in beef and consequences for meat quality. In: Indicators of milk and beef quality, EAAP Publications $\mathrm{n}^{\circ} 112$ (editors JF Hocquette and S. Gigli), Wageningen Academic Publishers, 2005, pp 151-62. 